\title{
Using a User Experience Evaluation Framework for eModeration
}

\author{
Cornè J van Staden \\ School of Computing \\ UNISA \\ Florida, South Africa \\ vstadcj1@unisa.ac.za
}

\author{
Prof Judy A van Biljon \\ School of Computing \\ UNISA \\ Florida, South Africa \\ vbilija@unisa.ac.za
}

\author{
Prof Jan H Kroeze \\ School of Computing \\ UNISA \\ Florida, South Africa \\ kroezjh@unisa.ac.za
}

\begin{abstract}
The use of eModeration (of examination scripts) can improve the efficiency of the examination moderation, while simultaneously lowering the risk of misplacing or delaying the moderation process. Despite the potential benefits of using an eModerate system in terms of optimising examination procedures, the implementation and application of such online moderation systems in the South African context is limited. Various factors could be contributing towards the resistance to the implementation and adoption of eModerate systems in higher education institutions. These factors include human factors as well as technical and organisational resistance to change. This study focuses on the human factors involved in eModeration (user experience) and attempts to answer the following research question: How can the User Experience Evaluation Framework for eModeration be utilised within the context of higher education institutions in South Africa? The research used a Design Science Research methodology, which included the design, development as well as testing of the User Experience Evaluation Framework for eModeration. This paper will report on identified issues pertaining to the User Experience Evaluation Framework for eModeration during the evaluation phase. The research was conducted at Midrand Graduate Institute (MGI), now trading as Pearson Institute of Higher Education, a private higher education institute in South Africa. The data generation methods included interviews with eModerators from different faculties within a private higher education institution. This paper makes a theoretical contribution to this area of study by identifying the problems that users might have with the implementation of the User Experience Evaluation Framework for eModeration as well as providing some insights into the user experience of eModerators.
\end{abstract}

Keywords - eModeration, eModerators, user experience, functionality, effectiveness, efficiency

\section{INTRODUCTION}

Manual paper-based moderation is still widely used at academic institutions in South Africa but the manual process poses challenges in terms of cost and time $[4,5]$. An additional challenge can be found in the intensive management process associated with manual moderation. The challenges that educators at academic institutions experience with regards to paper-based moderation call for an investigation into moderation processes. "Midrand Graduate Institute (MGI), a private higher education institution (PHEI) in South Africa, reviewed their examination and moderation practices and realised" [37:1] that there was a need to revise "assessment practices and structures" [37:1]. MGI decided after the investigation to replace the paper-based moderation process with an electronic process [5].

The role of information communication technology has changed over the years. The researcher recognised an emerging research trend and direction in the user experience of electronic moderation, which lead to an investigation of some of the challenges that educators might experience with finding, evaluating and using electronic moderation systems. Crucially, the researcher found that no user experience evaluation framework for eModeration existed at the time of this research. This work importantly addresses this lack of framework and contributes to the body of knowledge by proposing and creating a User Experience Evaluation Framework for eModeration, that can be used by educators to assess how appropriate an eModerate system is for their needs [4], with conceivable limitations to electronic moderation including connectivity, access to the internet and bandwidth.

Within "the context of this research, electronic moderation, also referred to as eModeration, involved a process of moderating examination scripts online" [37:1]. The following definition of eModeration was used in this study: "eModeration can be defined as the electronic moderation [quality assurance/critical reading] of summative examination scripts by external moderators in a virtual learning environment called eModerate" [22:3]. Several different types of users were involved in the electronic moderation process, such as examiners, moderators and the deans of the various faculties. The deans of the faculties managed the moderation process [37]. The user experience that was investigated was that of the eModerators. This paper reflects on the issues identified by 
eModerators concerning the proposed User Experience Evaluation Framework for eModeration. The study used the following question: How can the User Experience Evaluation Framework for eModeration be used within the context of higher education institutions in South Africa? This question explored in this paper by first offering a literature review that focuses on the concepts, followed by a discussion about the research approach and execution, then followed by a summary and discussion of the research results and lastly the conclusion.

\section{LITERATURE REVIEW}

Given the newness of eModeration in the South African context, "it is necessary to consider the difference between manual moderation and electronic moderation" [37:1]. After a short discussion about electronic moderation this paper will consider the relationship between electronic moderation and user experience.

\section{A. Manual moderation and electronic moderation}

The term moderation refers to the process of ensuring that internal and external verification took place, moderation further ensure that those being assessed are assessed in an "fair, valid, reliable and practicable" manner [1:7]. Moderation ensures "that the examiners or markers are making similar judgments about the learners' performance" [37:2]. The moderation task involves a process of ensuring that assessors used comparable assessment standards by reviewing a sample of learners' work that has been marked $[2,3]$. Manual moderation "will normally be paper-based. When the moderation is done electronically (online) the assessments and related functionalities are made available through an electronic application" [37:2].

The term 'eModeration process' often refers to feedback on assessments provided by the lecturer (who acts as an eModerator) to a student, using an electronic platform $[3,6,7$, 8]. An eModeration system provides a user interface through which the user can upload marked examination scripts electronically for moderation [4]. The marked paper-based examination scripts are scanned and uploaded on to the eModerate system. The eModerator then logs into the eModerate system and downloads the marked scripts. Using electronic assessment tools, feedback is provided in the form of comments or changed marks indicated in a different colour from that used on the originally marked script. After eModeration is complete the eModerator will upload the moderated scripts for review. The electronic moderation process is meant to speed up both the delivery of examination scripts to the moderator, as well as the return of quality assured eModerated scripts to the institution [5]. The eModeration process should limit the risk of losing scripts by making the tracking of scripts and provision of feedback easier during the moderation process. The difference between manual paperbased and eModeration is the way in which the grading of students' assessments is presented [9].

A few researchers have published work on eModeration assessment practices [9, 10,11], but very little information could be found about the eModeration of examination scripts in the South African context. Given the possible benefits of using eModeration it is necessary to investigate this gap in knowledge concerning the issues that influence the user experience of eModeration systems.

In the context of this research the user who moderates examination scripts electronically and provides feedback on the assessment using an eModerate system will be referred to as the eModerator.

\section{B. User experience and eModeration}

User experience refers to how the user feels while interacting with a product: for example, a web application [12, $13,14,37]$. User experience also includes the user's overall interaction with the system and perceptions resulting from the interaction $[12,15]$. For the purpose of this paper, Hassenzahl and Tractinsky's [16] definition of 'user experience' will be used. Reference [16] adopted in [37] and in study indicates that user experience is the consequence of the following components: system (characteristics of a system), context (environment in which the user operates) and the user's internal state such as needs and motivation.

The researcher understand user experience "as a concept where the user is placed at the focal point of design and development, instead of the system alone, and is made up of usability, functionality, context, system and the user's internal state" [37:2].

Usability includes the user's experience when interacting with the system as well as the usability of systems [14]. This study supports definitions of usability [18] that focus on how efficiently the users use the system, how fast the users learn to use the system, the extent to which the user prefer to use the system, how memorable the experience is and how often does the system produce errors [37]. The researcher subscribe to the relationship described by [19] "where usability is a component of user experience" [37:2]. This paper will not focus on "an in depth discussion of the relationship between usability and user experience" [37:2]. It is, however, important to take note that usability is an essential part of interactive systems: for example, online moderation systems.

User experience is influenced by the interaction between the following constructs: system, context and the user's internal state $[20,37]$. By measuring user experience - specifically the user's internal state, the user's perceived experience of the system's “instrumental (usability) and non-instrumental (appeal) qualities associated with the design process" [37:2] one can ascertain the extent of the impact of these qualities on the user's emotional reaction and acceptance or experience of eModeration [19, 20, 21, 22].

The researcher mapped user experience constructs of different authors [13, 16, 17, 23, 24] against eModeration to guide the design and development of the questionnaire that was used to "determine which user experience constructs would be relevant" [37:2] to a User Experience Evaluation Framework for eModeration. Key constructs identified through the literature review, and taken into consideration in terms of eModeration user experience for this study include: system (eModeration, web applications and connection), context (eModeration in PHEI's) and user (eModerator and manager) [5]. Existing user experience frameworks focus on the 
purchasing of products (eCommerce) or searching for information, and not on eModeration web pages. The researcher made use of the concepts and principles used in the user experience framework of [21] and [25] to support the conceptual User Experience Evaluation Framework for eModeration. Following this, the User Experience Evaluation Framework for eModeration was then presented, designed and developed [4]. The researcher then needed to evaluate the User Experience Evaluation Framework for eModeration using eModerators working within the context of PHEIs in order to ensure that the framework will support academic processes associated with assessment practices.

\section{RESEARCH APPROACH}

\section{A. Research design}

The study used the Design Science Research approach. The Design Science Research approach involves following a process of six steps and different cycles: relevance, design and rigour [27, 37]. During the relevance cycle the researcher conducts a literature review as well as context analysis in order to develop a conceptual framework. The design and development cycle focuses on the development and formative evaluation of the artifact using an iterative design process. The rigour cycle focuses on the generation of design principles. The Design Science Research approach was chosen because of the guidelines that is provides to do research [26]. As an approach, Design Science Research was deemed appropriate for the design and development of a user experience evaluation framework for eModeration, because it makes use of the evaluation and iteration phases.

In order to conduct a proper evaluation of an artifact it is necessary to structure evaluation activities with corresponding evaluation criteria using the concepts of evaluation patterns associated with Design Science Research artifacts as proposed in literature review [33]. As previously mentioned, the Design Science Research process incorporates a design-evaluateconstruct-evaluate pattern as shown in Fig. 1 [33]. Some researchers suggest that the Design Science Research process should use ex ante evaluations to validate the design of the artifact and the ex post evaluations to confirm that the artifact in use is solving the problem [33, 34, 35]. The evaluation as recommended Sonnenberg and Vom Brocke [33] was adapted for the use of this study. Fig. 1 demonstrates not only the evaluation activities of the Design Science Research process (top), but also the application thereof in this study (bottom).

For the purpose of this paper attention will only paid to the design and development of the artifact during the third evaluation and iteration phase (ex post evaluation). The literature review was done during evaluation and iteration one. and was then used to identify and understand the relevance of the research problem. During the second evaluation and iteration the researcher determined "which user experience constructs would be relevant to the design and development of a user experience evaluation framework for eModeration" [4:356]. The purpose of the third evaluation and iteration phase was to determine whether the artifact met the functionalities and requirements established during design and development [30].
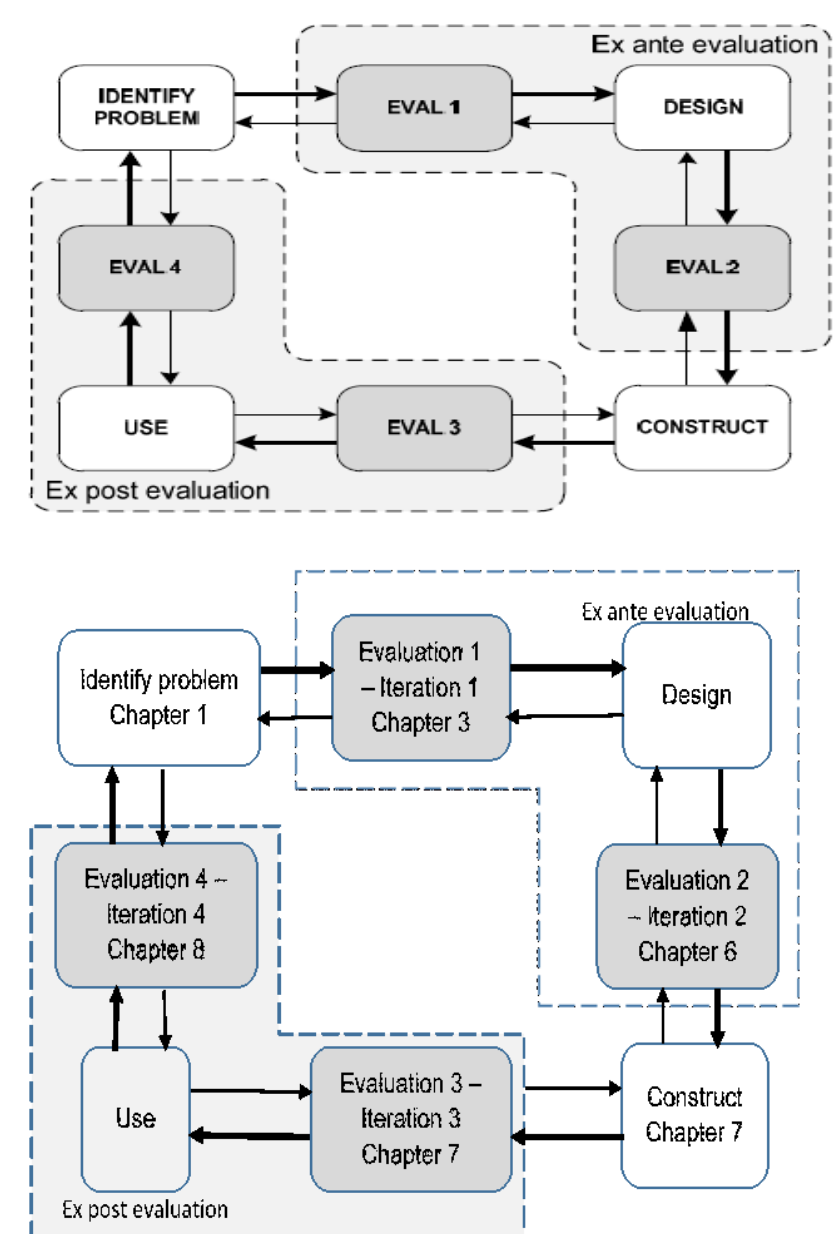

Figure 1 Evatuatition activities of Design Science Research process [34]

During the third evaluation and iteration the designed User Experience Evaluation Framework for eModeration was presented to eModerators and interviews were conducted in order to refine the designed framework.

\section{B. Research context}

Midrand Graduate Institute's (MGI) eModerate system was utilised in the design and development of the initial conceptual framework. The electronic moderation system allowed users to moderate the scanned examination scripts electronically using different eAssessment tools. The eModerators downloaded the examination scripts, moderated and uploaded the moderated scripts accompanied with reports for the deans or person responsible for managing the moderation of examination scripts. Three entities were involved in the moderation process at MGI:

- the lecturers who scored the examination scripts;

- the deans who arranged for the marked scripts be uploaded on to the eModerate system, who received moderators reports and who provided feedback to lecturers; and

- the eModerator who moderated the marked examination scripts, and acted as an external assessor or second examiner. 


\section{Sampling strategy}

The private higher education institution had 70 moderators, but only 30 wished to participate in the survey (evaluation and iteration two). Purposive sampling was followed to select 2 participants from each faculty. An invite was send to 3 participants in each faculty of which 2 per faculty agreed to take part in the interviews. A sample of 6 out of the 30 eModerators agreed to be participate in evaluation and iteration three where interviews were used. The eModerators based in the following faculties participated in the survey: Information Technology, Commerce, Creative Arts and Science.

\section{Research strategy}

A case study strategy was used to generate data, which involved collecting detailed information interviews. The structured interview was designed using open-ended questions as well as some Likert scale items. The open-ended questions were used to determine the eModerators views about the User Experience Evaluation Framework for eModeration. The qualitative data collected was useful for identifying themes because it provided a better understanding of the research problem [28, 29]. The evaluation of the User Experience Evaluation Framework for eModeration aided in validating the artifact within the context of the identified problem. The evaluation assisted within determining the relevance and applicability of such a framework, which academics could use to evaluate possible eModerate systems, for example, Moodle or google docs.

A diagrammatical representation of the designed User Experience Evaluation Framework for eModeration, together with a detailed explanation of each construct presented in a tabular format, was emailed to the interviewees before the interview along with an information sheet, see Fig. 2.

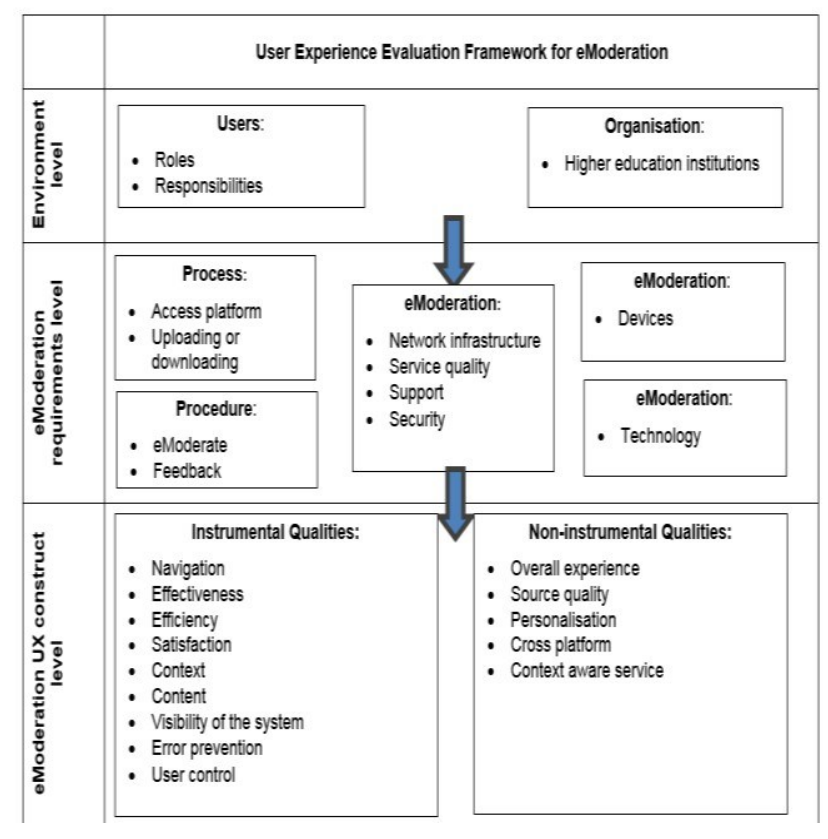

Figure 2 User Experience Evaluation Framework for eModeration
The interview was designed in such a way that the researcher could gain a deeper understanding of issues concerning user experience which might influence the adoption of eModeration and use these insights to inform e design of the framework. The structured interview was designed to determine the following:

- whether the user experience constructs identified by the survey were satisfactory for the design of the User Experience Evaluation Framework for eModeration;

- whether the identified levels (environment, eModeration requirements and user experience constructs) were adequate for measuring the user experience of eModeration;

- which of the user experience constructs, that had been identified as issues by participants, should be added or removed from the framework;

- whether the designed artifact would be easy enough to use;

- whether the framework made it possible to comprehend the essence of the modelled concepts;

- whether the framework was general enough to address a variety of problems;

- whether the framework was applicable and solved the research problem;

- whether the purpose of the constructs was clearly explained: for example, the operations or use of each element and interaction or flow between constructs;

- whether the framework was complete, by evaluating the users' satisfaction level with respect to completeness, effectiveness, satisfaction with requirements and if the problem was solved.

Various aspects need to be taken into consideration when generating evaluation criteria. The evaluation criteria to be used for the evaluation of artifacts should be independent of an artifact type in order to determine internal consistency, broad purpose and scope [31]. One set of evaluation criteria should focus on the relevance of the artifact, for example, is an artifact expected to be applicable in practice [32]. Some researchers also indicates that artifacts should be evaluated using criteria relevant to the requirements of the context in which the artifact is implemented: for example, in terms of "functionality, completeness, consistency, accuracy, performance, reliability, usability, fit with the organisation and other relevant quality attributes" [26:85].

The feedback, comments and responses of the eModerators were used to refine the User Experience Evaluation Framework for eModeration. A thematical analysis approach was used to identify themes [36]. An initial coding structure was identified and themes emerged from the data across the subset of data. Common themes across the data were grouped together and were then defined in more detail and named accordingly. The participants had to rate the three levels indicated in the User Experience Evaluation Framework for eModeration based on the principles used for the evaluation of artifacts and 
frameworks namely: simplicity, generality, exactness, clarity, completeness and relevance. The following Likert scale was used: "good", "needs improvement" or "not adequate". Participants could also comment on why they, for example, indicated that a construct needed improvement.

\section{FINDINGS}

The following section reports on the research findings based on the interviews with eModerators. The insights that were gained during the interviews were used to refine the User Experience Evaluation Framework for eModeration.

Based on the first questions all six of the eModerators agreed that there was no need to add an extra level to the framework. Participant D indicated that: "making the model too complex might lead to confusion and slow down the process flow", while Participant F indicated that they: "would not add another level as the three levels cover all aspects of eModeration". An issue that did emerge in one of the participant's responses concerning the framework was how the word 'context' should be understood and where it should be included. To resolve the issue the researcher added more details to the evaluation criteria associated with 'context'. The evaluation criteria will allow academic institutions that are investigating eModerate systems to check whether or not the system provides the functionality required to accommodate the unique characteristics of specific module assessments: for example, theoretical versus practical modules.

Table 1 reflects the qualitative feedback received from eModerators concerning whether or not other types of institutions could benefit from using the framework and if any additional users should be added to the framework. Two themes emerged from the questions posed to the interviewees concerning the environment level. The first was that the framework could be used in more than one type of institution for more than one type of purpose, not only for the moderation of examination scripts. The second theme that emerged was that IT support should be added under 'users of the system'. Letters A-F were assigned to each of the eModerators who where interviewed and used to maintain their anonymity.

TABLE I. FEEDBACK FROM EMODERATORS ABOUT THE ENVIRONMENT LEVEL

\begin{tabular}{|l|l|}
\hline \multirow{2}{*}{ Participant } & \multicolumn{1}{|c}{ Can the framework be used at any other institution? } \\
\cline { 2 - 3 } A & $\begin{array}{l}\text { "I think the criteria could be applied to any system } \\
\text { as both a quality assurance and user experience } \\
\text { evaluation framework." }\end{array}$ \\
\hline C & $\begin{array}{l}\text { "No, I think it is customised for higher education } \\
\text { institutions only." }\end{array}$ \\
\hline D & $\begin{array}{l}\text { Public and private HEIs will benefit as well as } \\
\text { Colleges, schools and other academic institutions. }\end{array}$ \\
\hline F & $\begin{array}{l}\text { Indicated that it can be used in any institution that is } \\
\text { involved in moderation }\end{array}$ \\
\hline D & $\begin{array}{l}\text { If too mumber of users identified adequate? } \\
\text { confusion or slow down the process flow. }\end{array}$ \\
\hline A & $\begin{array}{l}\text { The number of users are adequate, but the person } \\
\text { recommended that an IT support person be added as } \\
\text { a crucial role. }\end{array}$ \\
\hline
\end{tabular}

All six of the participants agreed that the environment level was well designed with respect to simplicity, generality, clarity and relevance. Only one participant wanted specific details to be provided under technology.

As a result IT support being added as a user with roles and responsibilities under the environment level, the participants recommended that IT support should also be added as a requirement under the eModeration requirement level. As Participant A rightfully indicated: "IT support plays a crucial role in the job of the eModeration Systems Operator". It was also suggested that in the event that an institution cannot afford an additional user, the roles and responsibilities should then be allocated to the eModeration Systems Operator.

Participants agree that the evaluation criteria discussed under the eModeration requirements level were clear and comprehensive. Under the generality of the requirements level one of the participants commented that where technology was mentioned Moodle and Google docs should possibly be removed. A decision was made not to remove Moodle and Google docs because no off-the-shelf software could be found, so the two examples that were provided should be understood as guidelines of software institutions could use perform the task of eModeration.

Lastly, the eModerators were asked to comment on the eModeration User Experience construct level, which was divided into two categories: instrumental and non-instrumental qualities. All of the participants agreed that the identified constructs were relevant, clearly explained, comprehensive and complete. One participant commented on simplicity saying that: "the entire process is simple and effective. The first solution to remote moderation I encountered that really works well and smoothly. Congratulations, this is a breakthrough for HEIs and other academic institutions in SA". Under relevance one of the participants said that the framework is: "perfect for external moderation! Very useful for internal moderation as well to keep track and record of each semester's examination results and moderation".

\section{CONCLUSION}

In conclusion, feedback from the eModerators proved to be beneficial in terms of refining the User Experience Evaluation Framework for eModeration. IT support was added as an extra user role along with its respective responsibilities, and was also added to the requirements level along with the appropriate evaluation criteria. Overall, the eModerators were satisfied with the instrumental and non-instrumental qualities and did not indicate a need to make changes to the eModeration user experience level. Therefore, it can be concluded that the evaluation of the User Experience Framework for eModeration provided useful feedback for refining the framework.

\section{ACKNOWLEDGMENT}

I would like to thank the participants for their time and valuable feedback. We also recognise UNISA for financial assistance. 


\section{REFERENCES}

[1] SAQA. 2015. "The South African Qualifications Authority: National Policy and Criteria for Designing and Implementing Assessment for NQF Qualifications and Part-Qualifications and Professional Designations in South Africa," http://www.saqa.org.za/list.php?pageNum rs=0\&totalRows rs $=24 \& \mathrm{e}=\mathrm{P}$ olicy

[2] H. Coates, "Defining and monitoring academic standards in Australian higher education," Australian Council for Educational Research (ACER), Higher Education Management and Policy, Vol. 22, 1, 2010, pp.1-18.

[3] P. Bridge and R. Appleyard, "A comparison of electronic and paperbased assignment submission and feedback," British Journal of Educational Technology, Vol. 39, 4, DOI: 10.1111/j.14678535.2007.00753X, 2008, pp. 644-650.

[4] C.J. Van Staden, J.A. Van Biljon and J.H. Kroeze, "Adopting eModeration: Understanding the user experience in the organisation," $8^{\text {th }}$ European Conference on IS Management and Evaluation. ECIME 2014 Conference, [Ghent, Belgium, September 2014], ISBN 978-1-91030941-4, 2014.

[5] C.J. Van Staden, "IT moderation going green!," SAICSIT'10. Conference, [Bela Bela, Limpopo, South Africa, September 2010], pp. 1-3, UNISA Production Printers, 2010.

[6] A. Morgan, "eModeration: contextualising online learning in undergraduate nurse education," Asian Journal of Nursing, Vol. 11, 1, 2008, pp. 48-53.

[7] G. Salmon, "E-moderating: The key to teaching and learning online," London: Kogan Page, 2003.

[8] P. Vlachopoulos, "The nature of e-moderation in online learning environments," LICK 2008 Symposium Edinburg: [Napier University / TESEP], http://www.napier.ac.uk/transform, 2008, pp. 48-57

[9] J. Greatorex, "Moderated e-portfolio evaluation," Evaluation and Validation Assessment Directorate UCLES. http://www.ucles.org.uk/ 2013.

[10] L. Addie, "Towards a theoretical framework for online professional discussions," Trattoda http://eprints.qut.edu.au/46921, 2014.

[11] D. Clow, "Digital residents and digital tourists,". Doug Clow's Imaginatively-Titled Blog. New Technology in Higher Education, http://dougclow.wordpress.com/2009/08/06/digital-residents-and-digitaltourists/, 2009.

[12] M. Kuniavsky, "Smart things: Ubiquitous computing user experience Design," Burlington, MA: Elsevier, ISBN: 978-0-12-374899-7, 2010.

[13] K. Paluch, "User experience design blog: Commentary on strategy and design of interactive products," http://www.montparnas.com/articles/what-is-user-experience-design/ 2006.

[14] Y. Rogers, H. Sharp and J. Preece, J. "Interaction Design," 3rd ed., Chichester, West Sussex, United Kingdom: John Wiley \& Sons Ltd, 2011.

[15] T. Tullis, and B. Albert, "Measuring the user experience," Burlington: Morgan Kaufmann, 2008.

[16] M. Hassenzahl, and N. Tractinsky, "User experience - a research agenda," Behaviour and Information Technology, Vol. 25, 2, 2006, pp. 91-97.

[17] ISODIS9241-210, "Ergonomics of human systems interaction - Part 210: Human-centred design for interactive systems (formerly known as 13407)," International Organisation for Standardisation. https://www.iso.org/obp/ui/\#iso:std:iso:9241:-210:ed-1:v1:en, 2010.

[18] J. Nielsen, and H. Loranger, "Web usability," Berkeley, CA: New Riders Press. http://www.nngroup.com/books/prioritizing-webusabilityl, 2006.
[19] H. Väätäjä, T. Koponen and V. Roto, "Developing practical tools for user experience evaluation," A Case from Mobile News Journalism. ECCE'09 European Conference on Cognitive Ergonomics, [Finland] ACM, http://dl.acm.org/citation.cfm?id=1690539, 2009, pp. 311-318.

[20] M. Hassenzahl, and N. Tractinsky, "User experience - a research agenda," Behaviour and Information Technology, Vol. 25, 2, 2006, pp. 91-97.

[21] S. Mahlke, and M. Thuring, "Studying antecendents of emotional experiences in interactive contexts," In Proceedings of CHI 2007 Conference on Human Factors in Computing Systems, [San Jose: CHI], Vol. 1, 2007, pp. 915-918.

[22] J. Nielsen, "User satisfaction vs. performance metrics," N. N. Group, Ed. [October, 8, 2012] http://www.nngroup.com/articles/satisfaction-vsperformance-metrics/, 2012

[23] R. Rubinoff, "How to quantify the user experience," Chigarge: APA, 2009.

[24] V. Roto, "Web browsing on mobile phones - characteristics of user experience," Doctoral Dissertation. Helsinki: University of Technology, 2006.

[25] K. Schulza, and H. Kromker, "A framework to measure user experience of interactive online products," In Proceedings of MB'10 Conference, [Eindhoven, Netherlands, August 24-27, 2010], ACM, New York, NY, 14,. DOI: $10.1145 / 193144.1931358,2010$.

[26] A.R. Hevner, S.T. March, J. Park. And S. Ram, "Design science in Information Systems Research," MISQuarterly, Vol. 28, 1, http://www.jstor.org/stable/25148625\%20Accessed\%20April\%2026, 2004, pp. 75-105.

[27] A. Hevner, "A three cycle view of Design Science Research," Scandinavian Journal of Information Systems, Vol. 19, 2, 2007, pp. 8792.

[28] L. Lazar, J. Feng H. and Hochheiser, "Research methods in HumanComputer Interaction," Glasgow: Bell \& Bain, 2010.

[29] J.W. Creswell, "Research design: Qualitative, quantitative, and mixed methods approaches," Los Angeles: Sage, 2009.

[30] T. Ellis and Y. Levy, "A guide for novice researchers: Design and development research methods," In Proceedings of Information Science \& Information Technology Education Conference [InSITE May 2, 2010],

http://citeseerx.ist.psu.edu/viewdoc/download?doi=10.1.1.170.2962\&rep $=$ rep1\&trype=pdf, 2010 .

[31] S. Aier and C. Fischer, "Criteria for progress of Information Systems design theories," Information Systems and E-Business Management, Vol. 9, 1, 2011, pp. 133-172

[32] M. Rossmann and I. Vessey, "Toward improving the relevance of Information Systems Research to practice: The role of applicability checks," MIS Quaterly, Vol. 32, 1, 2008, pp. 1-22.

[33] C. Sonnenberg and J. Vom Brocke, "Evaluations in the science of the artificial - reconsidering the build-evaluate pattern in Design Science Research," Design Science Research in Information Systems: Advancees in theory and practices, Vol. 7286, 2012, pp.381-397.

[34] S. Gregor, "The nature of theory in Information Systems," MIS Quaterly, Vol. 30, 2006, pp. 611-642.

[35] S. Gregor and D. Jones, "The anatomy of a design theory," Journal of the Association of Information Systems, Vol 9, 5, 2007, pp. 312-335.

[36] V. Braun and V. Clarke, "Using thematical analysis in psychology," Qualitatitive Research in Psychology, Vol 3, 2, 2006, pp. 77-101.

[37] C.J. van Staden, J.A. van Biljon, and J.H Kroeze, "eModeration: Towards a User Experience Evaluation Framework," SAICSIT 2015 Proceedings of the 2015 Annual Reseach Conference of the South African Institute of Computer Scientists and Information Technologists, [Stellenbosch, Western Cape, South Africa, September 28-30], ISBN: 978-1-4503-3683-3, 2015 